Article

\title{
Features of the Behavior of the Barocaloric Effect near Ferroelectric Phase Transition Close to the Tricritical Point
}

\author{
E.A. Mikhaleva ${ }^{1} \mathbb{D}$, I.N. Flerov ${ }^{1,2, * \mathbb{D}}$, M.V. Gorev ${ }^{1,2} \mathbb{D}$, V.S. Bondarev ${ }^{1,2} \mathbb{D}$ and \\ E.V. Bogdanov 1,3
}

1 Kirensky Institute of Physics, Federal Research Center KSC Siberian Branch, Russian Academy of Sciences, 660036 Krasnoyarsk, Russia; katerina@iph.krasn.ru (E.A.M.); gorev@iph.krasn.ru (M.V.G.);

vbondarev@yandex.ru (V.S.B.); evbogdanov@yandex.ru (E.V.B.)

2 Institute of Engineering Physics and Radioelectronics, Siberian Federal University, 660074 Krasnoyarsk, Russia

3 Institute of Engineering Systems and Energy, Krasnoyarsk State Agrarian University, 660049 Krasnoyarsk, Russia

* Correspondence: flerov@iph.krasn.ru

Received: 26 December 2019; Accepted: 16 January 2020; Published: 19 January 2020

\begin{abstract}
A detailed study of the effect of temperature and pressure on heat capacity, entropy and hysteresis phenomena near the ferroelectric phase transition in ammonium sulfate (AS) was performed. An analysis of experimental results within the framework of the phenomenological theory showed that taking into account the temperature-dependent part of the anomalous entropy leads to a significant increase in the barocaloric effect (BCE). The maximum values of extensive and intensive BCE near the tricritical point are outstanding: $\triangle S_{B C E}^{\max } \approx 85 \mathrm{~J} / \mathrm{kgK}, \Delta T_{A D}^{\max } \approx 12 \mathrm{~K}$ and can be achieved at low pressure $\sim 0.5 \mathrm{GPa}$.
\end{abstract}

Keywords: polymorphic phase transformation; phase diagram; order-disorder phenomena; entropy; barocaloric effect

\section{Introduction}

Solids showing significant caloric effects (CE) associated with the reversible change in the temperature, $\Delta T_{A D}$, and entropy, $\Delta S_{C E}$, under variation of the external field are considered as promising solid refrigerants in alternative cooling cycles [1-9].

Until recently, little attention was paid to the barocaloric effect (BCE) in ferroelectrics [10-14]. Nevertheless, it was found that, even at low pressures, the extensive, $\Delta S_{B C E}$, and intensive, $\Delta T_{A D}$, effects can significantly exceed the parameters of the electrocaloric effect realized in high electric fields $[10,13,14]$.

The most impressive results are associated with BCE in ferroelectrics: $\left(\mathrm{NH}_{4}\right)_{2} \mathrm{SO}_{4}$ (AS), and $\mathrm{NH}_{4} \mathrm{HSO}_{4}$ (AHS) $[11,15]$. The main reason for the significant $\mathrm{BCE}$ near the first-order phase transitions Pnam $\leftrightarrow P n a 2_{1}$ and $P c \leftrightarrow P 1$ in AS and AHS, respectively, is due to the large values of entropy changes, $\Delta S_{0}$, and baric coefficients, $d T / d p$.

At the same time, the transformations in these compounds differ from each other by their closeness to the tricritical point characterized, in particular, by the relation between the jump in entropy, $\delta S_{0}$, at the transition point, $T_{0}$, and its total change, $\Delta S_{0}$. In accordance with the $\delta S_{0} / \Delta S_{0}$ values equal to 0.50 and 0.93 in AS and AHS, respectively, the phase transition in the former ferroelectric is significantly closer to tricritical point, where $\delta S_{0} / \Delta S_{0}=0$. Nevertheless, the value of $\delta S_{0}$ was found to be more sensitive to pressure in AHS, reaching zero at $p_{T C P}=0.18 \mathrm{GPa}$, while for AS this value is about $0.36 \mathrm{GPa}$. 
Due to strong difficulties of the accurate measurements of the heat capacity of solids under pressure, the pressure dependencies of the temperature dependent anomalous entropy below the transition temperatures, $\Delta S(T, p)=\Delta S_{0}-\delta S_{0}$, are unknown in AS [11] and AHS [15]. This is the reason why BCE in both ferroelectrics was determined without taking into account the dependencies $\Delta S_{0}(T, p)[11,15]$.

Recently, calorimetric studies of the AS powder sample were performed by differential scanning calorimeter (DSC) [11]. In this case, it is difficult to obtain reliable information about the heat capacity $C_{p}(T)$, entropy $S(T, p=0)$ as well as their anomalous contributions associated with the phase transition. That is why the analysis of extensive BCE in AS was performed only taking into account the pressure dependences $T_{0}(p)$ and $\delta S_{0}(p)$ [11]. On the other hand, it is evident that the behavior and maximum values of BCE should be strongly sensitive to the dependencies $S(T, p)$ and $\Delta S_{0}(T, p)$.

In the present paper, to get reliable information on the dependencies $C_{p}(T, p=0), S(T, p=0)$, $\delta S_{0}(p)$ and $\Delta S_{0}(T, p=0)$ and as result on the extensive and intensive BCE, we performed studies of single crystal AS using an adiabatic calorimeter as the most accurate and sensitive calorimetric method. Such measurements avoided distortion of the temperature dependence of the anomalous entropy and smearing of its jump which was determined by the method of quasistatic thermograms. On the same sample, the pressure dependencies $T_{0}(p)$ and $\delta S_{0}(p)$ were determined using differential thermal analysis under pressure.

For phase transitions of the first order close to the tricritical point, the pressure behavior of both entropies can be restored analyzing the dependencies $S(T, p=0), \Delta S_{0}(T, p=0), \delta S_{0}(p)$ and $T_{0}(p)$ in the framework of the Landau phenomenological theory [16-18]. Such an analysis is impossible for AHS due to the almost complete absence at atmospheric pressure of temperature-dependent anomalous heat capacity below the transition $P c \leftrightarrow P 1$ [19]. In the case of AS, the relatively small value of $\delta S_{0} / \Delta S_{0}$ and the wide temperature range of the existence of $\Delta S(T, p=0)[11,20]$ suggest the applicability of the thermodynamic theory to the description of the transformation Pnam $\rightarrow$ Pna $_{1}$.

\section{Experimental Methods}

AS single crystals were grown at $40{ }^{\circ} \mathrm{C}$ from an aqueous solution of powder AS. XRD examination showed an absence of the secondary phases and revealed an orthorhombic symmetry consistent with the space group Pnam $(Z=4)$ with the expected lattice parameters [21].

Heat capacity measurements were performed on a single crystal with dimensions $5 \times 5 \times 2.5 \mathrm{~mm}^{3}$ by means of a homemade automated adiabatic calorimeter [22]. The inaccuracy in the heat capacity determination did not exceed $(0.3-0.5 \%)$ over the whole temperature range investigated $(80-280 \mathrm{~K})$. Discrete and continuous heating was used to measure the heat capacity of the "sample + heater + contact grease" system. In the former case, the calorimetric step was varied from 0.5 to $3.0 \mathrm{~K}$. In the latter case, the system was heated at rates $d T / d t=0.1-0.3 \mathrm{~K} / \mathrm{min}$. The heat capacities of the heater and contact grease were determined in individual experiments.

Using a homemade high-pressure chamber with multiplier [23], we performed differential thermal analysis (DTA) experiments to study the effect of hydrostatic pressure on temperature and entropy of the phase transition. Measurements were performed on a single crystal sample with dimensions of $2 \times 2 \times 2 \mathrm{~mm}^{3}$. A pressure-transmitting medium was a mixture of silicon oil and pentane. In order to measure temperature $T_{0}$ and $\Delta C_{p}$ related to the phase transition, we used the copper-constantan and highly sensitive differential copper-germanium thermocouples, respectively. The pressure was measured by a manganin resistive sensor.

\section{Results and Discussion}

The temperature dependence of the heat capacity $C_{p}(T)$ is shown in Figure 1a. Anomaly associated with the phase transition was observed at $T_{0}=222.18 \pm 0.10 \mathrm{~K}$. In the vicinity of $T_{0}$, investigations were carried out using a method of quasistatic thermograms with an average heating rate of $d T / d t=6.5 \times 10^{-3} \mathrm{~K} / \mathrm{min}$. Figure $1 \mathrm{~b}$ shows that the structural transformation is accompanied 
by enthalpy jump $\delta H_{0}=1450 \pm 45 \mathrm{~J} / \mathrm{mol}$ without smearing due to the high quality of the single crystal sample.

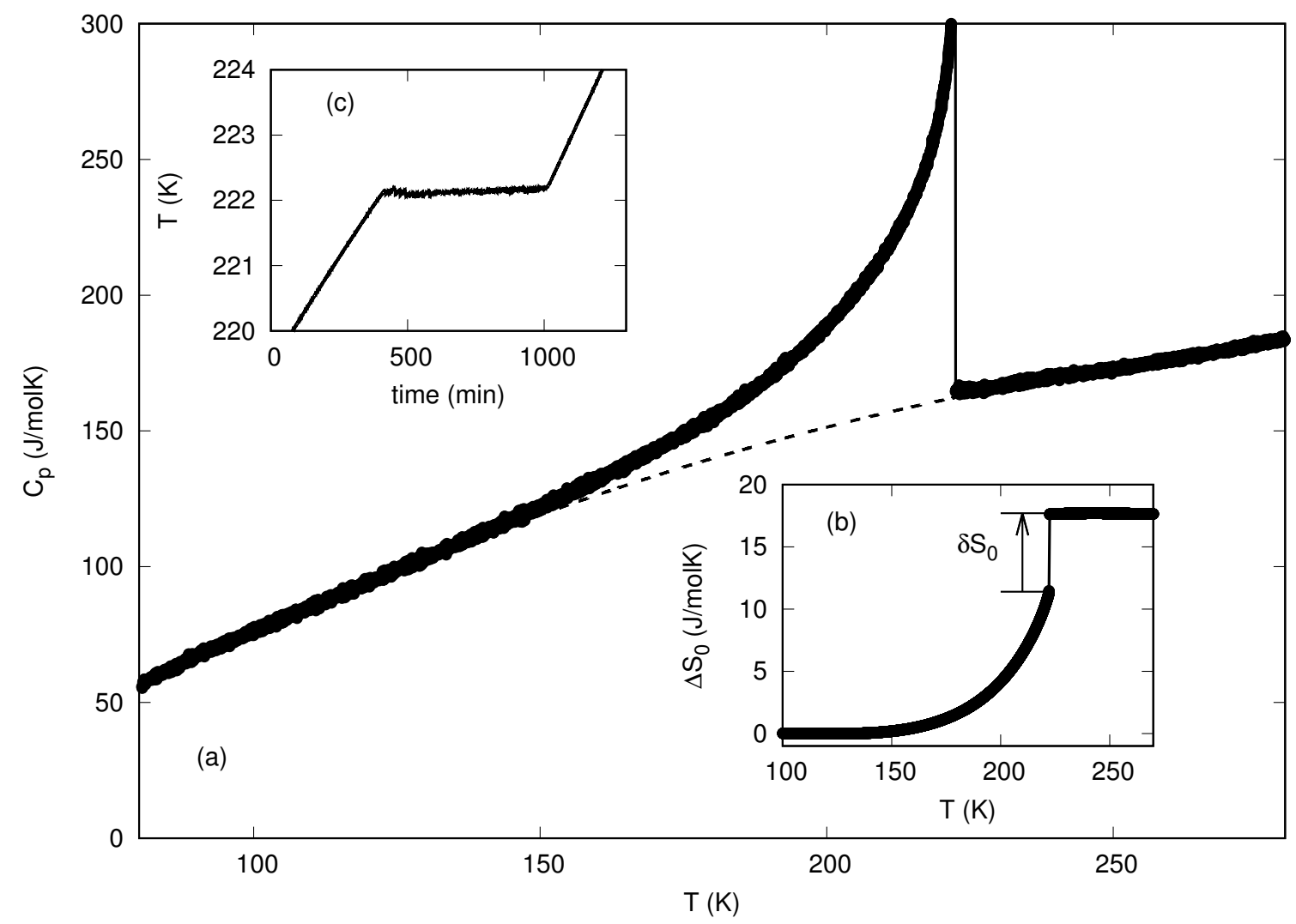

Figure 1. Temperature dependencies of (a) molar heat capacity and (b) anomalous entropy; (c) quasistatic thermogram near phase transition. The dashed line in (a) corresponds to the lattice heat capacity $C_{L}$.

Information on the integral entropy change of the phase transition was obtained by separating the anomalous, $\Delta C_{p}$, and lattice, $C_{L}$, contributions to the total $C_{p}$ using a simple model describing $C_{L}(T)$. The experimental data taken far from the transition point $(T<145 \mathrm{~K}$ and $T>230 \mathrm{~K})$ were fitted using a linear combination of Debye and Einstein terms $C_{L}=K_{D} C_{D}+K_{E} C_{E}$, where

$$
\begin{gathered}
C_{D}(T)=9 R\left(\frac{T}{\Theta_{D}}\right)^{3} \int_{0}^{\Theta_{D} / T} \frac{x^{4} \exp (x)}{(\exp (x)-1)^{2}} d x, \\
C_{E}(T)=3 R\left(\frac{\Theta_{E}}{T}\right)^{2} \frac{\exp \left(\Theta_{E} / T\right)}{\left(\exp \left(\Theta_{E} / T\right)-1\right)^{2}}
\end{gathered}
$$

and $K_{D}, K_{E}, \Theta_{D}, \Theta_{E}$ are fitting parameters. The average deviation of the experimental data from the smoothed curve does not exceed $0.5 \%$.

Figure $1 \mathrm{c}$ demonstrates the temperature behavior of entropy $\Delta S_{0}(T)$ associated with the phase transition in AS. Its total value $\Delta S_{0}=\int \Delta C_{p} / T=17.7 \pm 0.8 \mathrm{~J} / \mathrm{molK}$ agrees well with the value $3 R \ln 2=17.3 \mathrm{~J} / \mathrm{molK}$ following from a model consideration of the Pnam $\leftrightarrow P n a 2_{1}$ transition as an order-disorder process [24]. The entropy jump at $T_{0}, \delta S_{0}=\delta H_{0} / T_{0}=6.5 \pm 0.2 \mathrm{~J} / \mathrm{molK}$, is much smaller than the value $\delta S_{0}=8.5 \mathrm{~J} / \mathrm{molK}$ determined by the less accurate DSC method [11].

Thus, in accordance with $\delta S_{0} / \Delta S_{0}=0.37$, the transition in single crystal AS is much closer to the tricritical point in comparison with the powder sample $\left(\delta S_{0} / \Delta S_{0}=0.50\right)$.

In order to determine the hysteresis corresponding to the equilibrium thermal conditions, dependence of $T_{0}$ on the heating/cooling rate was studied in a wide range of the value $d T / d t=$ 
$\pm(2-16) \mathrm{K} / \mathrm{min}$ using a differential scanning microcalorimeter (Figure 2a). The dependencies $T_{0} \uparrow(d T / d t)$ and $T_{0} \downarrow(d T / d t)$ were found to be linear. At the rate of $d T / d t= \pm 8 \mathrm{~K} / \mathrm{min}$, thermal hysteresis $\delta T_{0} \approx 3.5 \mathrm{~K}$ is comparable to value $(3.5 \mathrm{~K})$ obtained at a scanning rate $\pm 10 \mathrm{~K} / \mathrm{min}$ [11]. Extrapolation of the $T(d T / d t)$ dependencies to $d T / d t=0$ allows one to obtain the real values of temperature of the phase transition $T_{0} \uparrow=222.5 \mathrm{~K}, T_{0} \downarrow=221.5 \mathrm{~K}$ and a strong decrease in the thermal hysteresis $\delta T_{0}=1 \mathrm{~K}$.
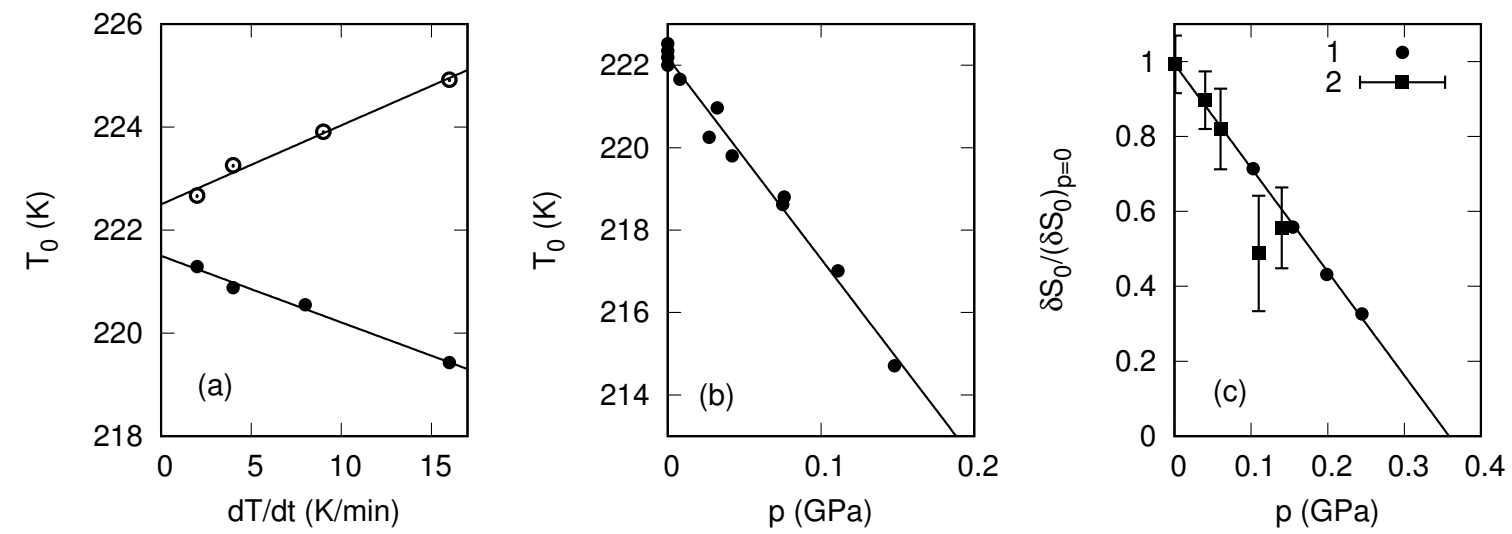

Figure 2. (a) dependence of the phase transition temperature $T_{0}$ on the heating/cooling rate; (b) temperature-pressure phase diagram; (c) entropy jump $\delta S_{0}$ for the first order transition at different hydrostatic pressures.

At ambient pressure, the anomaly of DTA signal was detected at about $T_{0}=223 \pm 1 \mathrm{~K}$ which agrees well with value observed during measurements of the heat capacity. Figure $2 \mathrm{~b}$ shows that an increase in pressure is accompanied by linear decrease in $T_{0}$ with the rate $d T_{0} / d p=-49 \pm 3 \mathrm{~K} / \mathrm{GPa}$, which is close to the value for powder sample obtained in Ref. [11].

Due to the limited sensitivity of DTA, the area under the DTA peak at $T_{0}$ represents the enthalpy $\delta H_{0}$ (entropy $\delta S_{0}$ ) jump at $T_{0}$. The value of $\delta S_{0}$ decreases with pressure and reaches zero at $\sim 0.36 \mathrm{GPa}$ that corresponds to the pressure of the tricritical point, $p_{T C P}$ (Figure 2c). On the other hand, it is unlikely that such a low pressure may affect the degree of disordering of structural elements in initial phase Pnam and as a result the total entropy change at the $P n a m \leftrightarrow P n a 2_{1}$ transformation $\Delta S_{0}$ remains constant.

Both BCE, $\triangle S_{B C E}$ and $\triangle T_{A D}$ in AS could be determined by the previously used method [25] using data obtained in the present work on the heat capacity, $T-p$ phase diagram, and temperature and pressure dependencies of $\Delta S_{0}$ and $\delta S_{0}$, respectively. However, as mentioned above, it was first necessary to restore the temperature dependencies of the anomalous entropy at various pressures taking into account the relationship between $\Delta S_{0}$ and the order parameter.

Despite the fact that polarization occurs during the phase transition in AS, there is no consensus on whether it is the main order parameter $[18,26,27]$. There are a number of models describing the phase transition in ammonium sulfate [18], but all of them do not fully take into account the interaction between the three types of tetrahedral groups and do not adequately describe the entire set of crystal properties based on the values of spontaneous deformation and polarization. For example, the model of an improper ferroelectric transition in AS [26] assumes that a certain parameter $\eta$ is the main order parameter connected bilinearly with polarization, which is not a critical parameter. Authors [26] introduce an ordering parameter $\eta$ of some atoms along the ferroelectric axis, which does not contribute to the polarization but plays the role of a trigger for the occurrence of the spontaneous polarization, into the potential function

$$
\Delta \Phi=\frac{1}{2} \alpha \eta^{2}+\frac{1}{4} \gamma \eta^{4}+\frac{1}{6} \delta \eta^{6}+\frac{1}{2} \chi P^{2}+f \eta P+\ldots
$$


As a result of a series of simple transformations, the potential (Equation (3)) takes the following form

$$
\Delta \Phi=A \eta^{2}+B \eta^{4}+C \eta^{6}
$$

where $A=A_{T}\left(T_{0}-T_{C}\right)+A_{T}\left(T-T_{0}\right)$ and $T_{C}<T_{0}$ is the Curie temperature at which the inverse susceptibility is zero.

In accordance with the equation of state, $\partial \Delta \Phi / \partial \eta=0$, the temperature dependence of the order parameter is as follows:

$$
\eta^{2}=\left[-B+\left(B^{2}-3 A C\right)^{1 / 2}\right] / 3 C
$$

and graphically in coordinates $\eta^{2}$ and $(T)$ looks like a parabola.

Given the relation between the jump in the order parameter at $T_{0}$ and the coefficients in Equation (5)

$$
\eta_{0}^{2}=-(B / 2 C)=-2 A_{T}\left(T_{0}-T_{C}\right) / B
$$

the equation of state (Equation (5)) can be represented as convenient for the analysis of experimental data

$$
\left[\eta^{2}-(2 / 3) \eta_{0}^{2}\right]^{2}=\left[(1 / 3) \eta_{0}^{2}\right]^{2}+A_{T}\left(T_{0}-T\right) / 3 C
$$

Here, the value $(2 / 3) \eta_{0}^{2}$ corresponds to the ordinate of the vertex of the parabola at $T^{*}$.

Analysis in the framework of thermodynamic theory can be performed taking into account the relation between the order parameter and entropy, as well as their jumps $[17,18]$

$$
\Delta S_{0}=\partial \Delta \Phi / \partial T=A_{T} \eta^{2} ; \delta S_{0}=A_{T} \eta_{0}^{2}
$$

Figure 3 a shows that, in a wide temperature range below $T_{0}$ at $p=0$, the dependence $\Delta S_{0}(T)$ is quite satisfactorily described in the framework of the phenomenological theory with the following parameters: $T^{*} \approx 223.1 \mathrm{~K}, T_{c} \approx 218.5 \mathrm{~K}, \delta S_{0} \approx 6.3 \mathrm{~J} / \mathrm{molK}$.

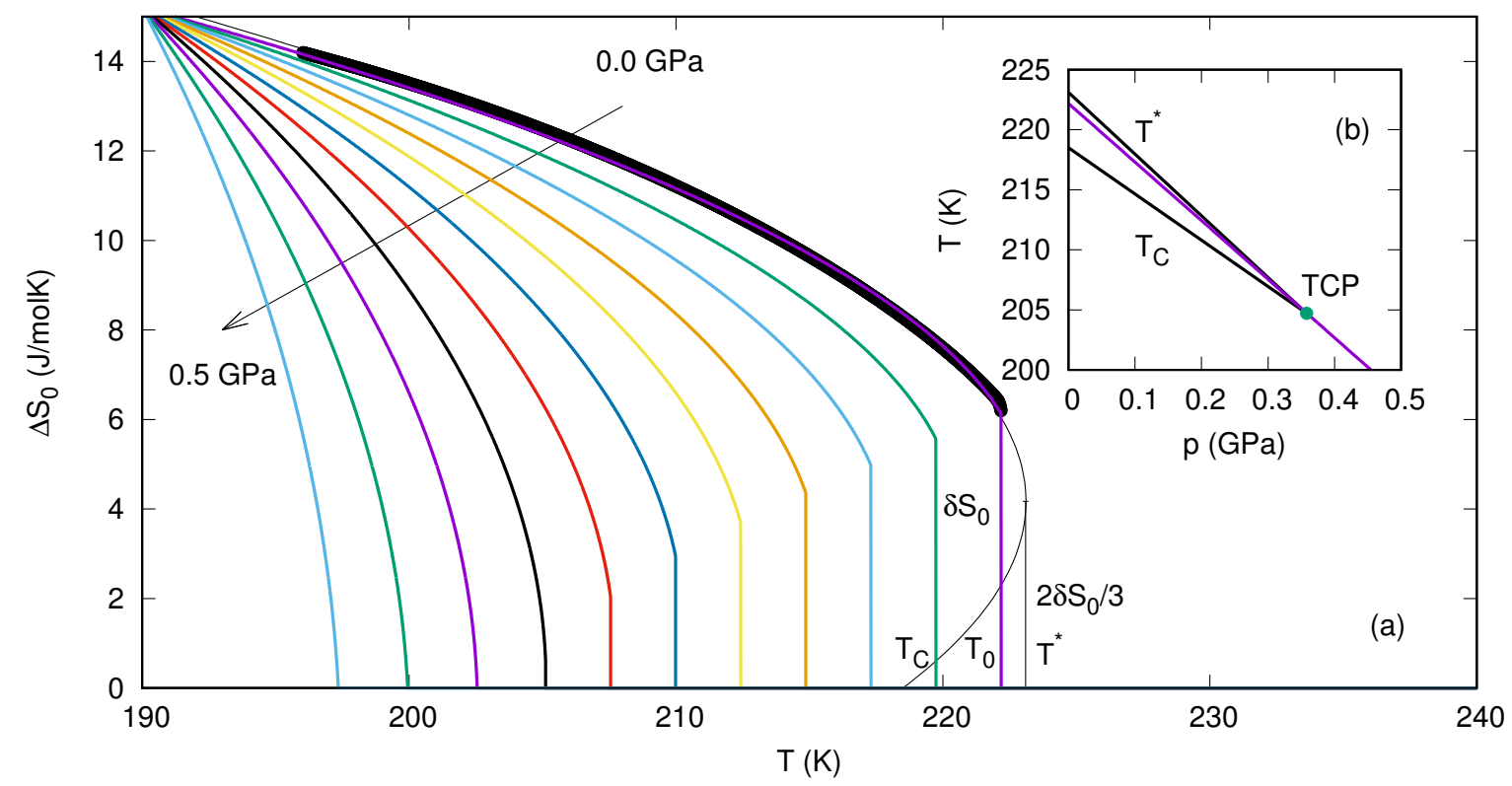

Figure 3. Temperature dependences of (a) anomalous entropy at various hydrostatic pressures;

(b) refined $T-p$ phase diagram.

The behavior of the function $\Delta S_{0}(T)$ under pressure up to $0.5 \mathrm{GPa}$ was restored taking into account the dependencies $T_{0}(p)$ and $\delta S_{0}(p)$ (Figure 3a). In this case, the $T-p$ phase diagram looks as shown in Figure 3b. An increase in pressure leads to a decrease in the difference between temperatures $T^{*}$ and $T_{C}$, which become equal to $T_{0}$ at $p=p_{T C P}$. As result at $p>p_{T C P}$, AS undergoes a second-order 
transformation close to the tricritical point. However, in this case too, Equations (5)-(7) remain valid for the analysis of the function $\Delta S_{0}(T)[16,17]$.

It was recently found that thermal expansion of the crystal lattice can play a significant role in the formation of BCE $[11,15,28]$. Indeed, in accordance with Maxwell equation $\left(\partial S_{L} / \partial p\right)_{T}=$ $-\left(\partial V_{L} / \partial T\right)_{p}$, the lattice contribution to the isothermal entropy change is proportional to volumetric thermal expansion coefficient $\beta_{L}$,

$$
\Delta S_{L}(T, p)=-\int_{0}^{p}\left(\partial V_{L} / \partial T\right)_{p} d p \approx-V_{m} \beta_{L}(T) p .
$$

The molar volume, $V_{m}$, and $\beta_{L}(T)$ are weakly dependent on pressure, which was experimentally established in Ref. [11].

Taking into account that AS is characterized by rather large positive value of $\beta_{L}(T)=(1.0-$ 1.5) $10^{-4} \mathrm{~K}^{-1}$ [11], temperature dependencies of the total entropy under different pressures were determined by summation of the lattice entropy, $S_{L}(T)$, and the anomalous contributions: $S(T, p)=$ $S_{L}(T, 0)+\Delta S_{L}(T, p)+\Delta S_{0}(T, p)$ (Figure 4a).
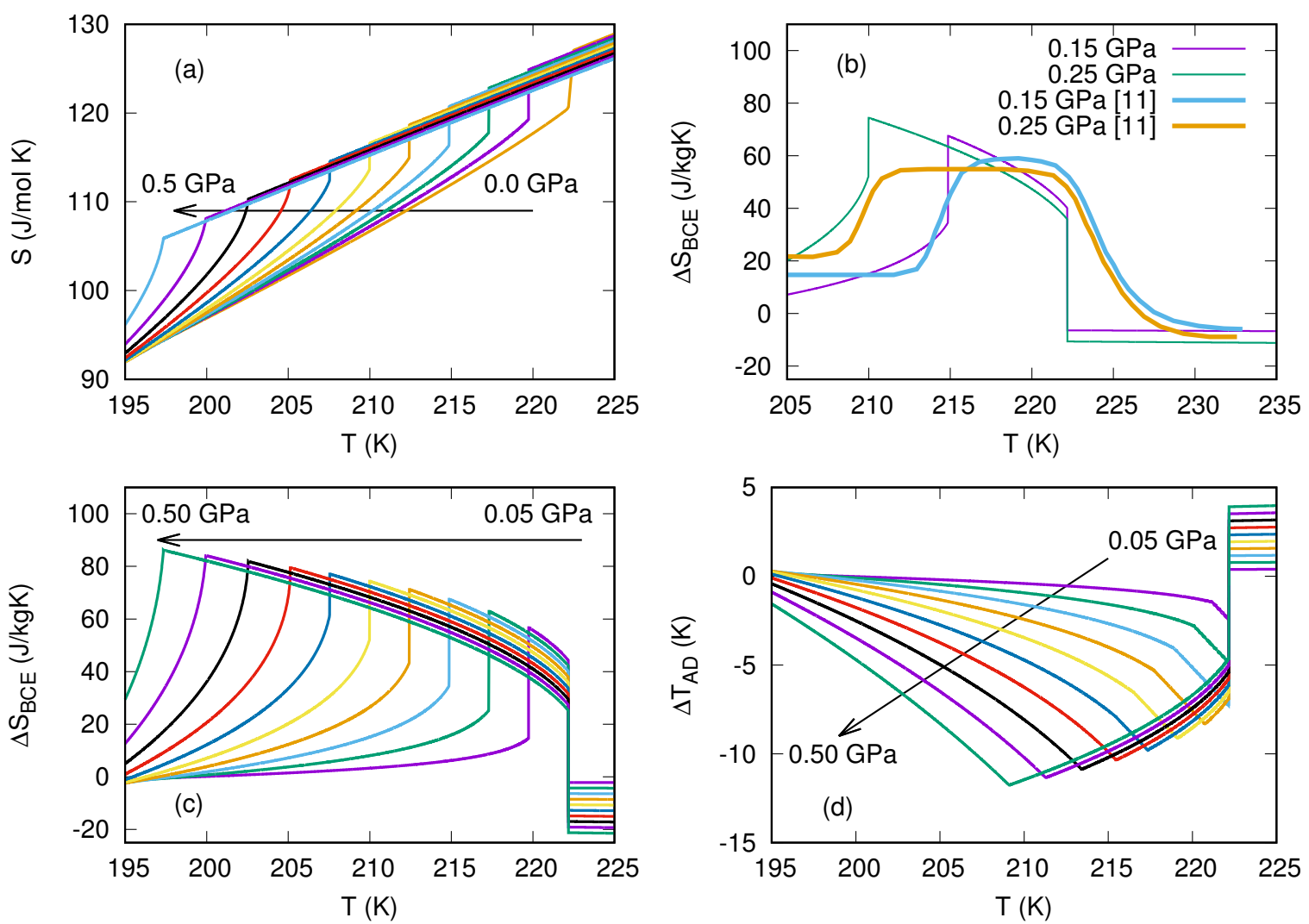

Figure 4. (a) temperature dependencies of total entropy at different pressures; (b) comparison of extensive BCE obtained at $p \leq 0.25 \mathrm{GPa}$ in this paper and in Ref. [11]; behavior of (c) barocaloric entropy and (d) adiabatic temperature change at pressures up to $0.5 \mathrm{GPa}$.

The extensive BCE was determined by analyzing the temperature dependencies of the total entropy at different pressures: $\Delta S_{B C E}=S(T, p)-S(T, p=0)$. Figure $4 \mathrm{~b}$ shows the data obtained in this paper and in Ref. [11] at relatively low pressures, $p \leq 0.25 \mathrm{GPa}$. It can be seen that taking into account the temperature-dependent part of the anomalous entropy correctly determined using the adiabatic calorimeter leads to a significantly different behavior of $\triangle S_{B C E}(T)$ and to an increase in its maximum value at the same pressure. Moreover, the restoration of the behavior of anomalous entropy under pressure made it possible to analyze the dependencies $\Delta S_{B C E}(T)$ and $\Delta T_{A D}(T)$ at pressures close to $p_{T C P}$ and higher (Figure $4 \mathrm{c}, \mathrm{d}$ ). To obtain correct information on the behavior 
of the intensive BCE, plots of $S(T, p)$ were analyzed based on the condition of constant entropy $S(T, p)=S\left(T+\Delta T_{A D}, p=0\right)$.

Due to the negative total coefficient $\beta$ in the region of the phase transition and $\beta_{L}>0$ [11], BCE in AS consists of two contributions: inverse, $\Delta S_{B C E}>0$, and conventional, $\Delta S_{B C E}<0$, at $T<T_{0}$ and $T>T_{0}$, respectively. This is the reason why the inverse extensive BCE cannot reach the maximum value, which is equal to the total entropy of the phase transition. However, even at $p=0.5 \mathrm{GPa}$, the maximum values of $\Delta S_{B C E}^{\max } \approx 85 \mathrm{~J} / \mathrm{kgK}$ and $\Delta T_{A D}^{\max } \approx 12 \mathrm{~K}$ are significantly higher in comparison with many materials undergoing phase transitions of various nature and considered as promising solid-state refrigerants $[1,3,7,8,11]$. Moreover, Figure 4 shows that, in accordance with Equation (9), the jump of the conventional $\mathrm{BCE}$ associated with the contribution of the crystal lattice is proportional to the pressure.

\section{Conclusions}

In conclusion, this letter demonstrates that the most correct information on BCE in materials undergoing phase transitions close to the tricritical point can be achieved by using heat capacity data obtained using adiabatic calorimeter. Analysis of these data for AS in the framework of the phenomenological theory, together with the dependencies $T(p), \delta S(p), T(d T / d t)$ determined in this paper, allowed: (1) to restore the magnitude and behavior under pressure of the entropy associated with the phase transition; (2) to build a detailed $T-p$ phase diagram showing the behavior of characteristic temperatures: $T^{*}, T_{C}$ and $T_{0}$ and, as a result, a change in hysteresis phenomena under pressure up to the tricritical point. It was shown that taking into account the temperature dependent anomalous entropy leads to a strong increase in the maximum values of $\Delta S_{B C E}^{\max }$ and $\Delta T_{A D}^{\max }$, as well as to a significant change in the temperature and pressure dependences of extensive and intensive BCE as compared to the case when only the behavior of the entropy jump was analyzed [11]. Even at low pressure $p \leq 0.5 \mathrm{GPa}$, AS demonstrates BCE parameters that are comparable and/or even exceed the same parameters for materials considered to be promising solid-state refrigerants $[1,3,7,8,11]$.

Author Contributions: Investigation, V.S.B. and E.V.B.; Conceptualization, I.N.F.; Formal analysis, E.A.M.; Writing-original draft preparation, M.V.G. All authors have read and agreed to the published version of the manuscript.

Funding: The reported study was supported by the Russian Science Foundation (project no. 19-72-00023).

Conflicts of Interest: The authors declare no conflict of interest.

\section{Abbreviations}

The following abbreviations are used in this manuscript:

AS ammonium sulfate

BCE barocaloric effect

DTA differential thermal analysis

XRD X-ray diffraction

\section{References}

1. Gschneidner, K.A., Jr.; Pecharsky, V.K.; Tsokol, A.O. Recent developments in magnetocaloric materials. Rep. Prog. Phys. 2005, 68, 1479-1539. [CrossRef]

2. Brown, J.S.; Domanski, P.A. Review of alternative cooling technologies. Appl. Therm. Eng. 2014, 64, $252-262$. [CrossRef]

3. Moya, X.; Kar-Narayan, S.; Mathur, N.D. Caloric materials near ferroic phase transitions. Nat. Mater. 2014, 13, 439-450. [CrossRef] [PubMed]

4. Plaznik, U.; Vrabelj, M.; Kutnjak, Z.; Malič, B.; Poredoš, A.; Kitanovski, A. Electrocaloric cooling: The importance of electric-energy recovery and heat regeneration. Europhys. Lett. 2015, 111, 57009. [CrossRef]

5. Kitanovski, A.; Plaznik, U.; Tomc, U.; Poredoš, A. Present and future caloric refrigeration and heat-pump technologies. Int. J. Refrig. 2015, 57, 288-298. [CrossRef] 
6. Liu, Y.; Scott, J.F.; Dkhil, B. Some strategies for improving caloric responses with ferroelectrics. APL Mater. 2016, 4, 064109. [CrossRef]

7. Mañosa, L.; Planes, A. Materials with giant mechanocaloric effects: Cooling by strength. Adv. Mater. 2017, 29, 1603607. [CrossRef]

8. Zarkevich, N.A.; Johnson, D.D.; Pecharsky, V.K. High-throughput search for caloric materials: The CaloriCool approach. J. Phys. D Appl. Phys. 2017, 51, 024002. [CrossRef]

9. Michaelis, N.; Welsch, F.; Kirsch, S.-M.; Schmidt, M.; Seelecke, S.; Schütze, A. Experimental parameter identification for elastocaloric air cooling. Int. J. Refrig. 2019, 100, 167-174. [CrossRef]

10. Mikhaleva, E.A.; Flerov, I.N.; Gorev, M.V.; Molokeev, M.S.; Cherepakhin, A.V.; Kartashev, A.V.; Mikhashenok, N.V.; Sablina, K.A. Caloric characteristics of $\mathrm{PbTiO}_{3}$ in the temperature range of the ferroelectric phase transition. Phys. Solid State 2012, 54, 1832-1840. [CrossRef]

11. Lloveras, P.; Stern-Taulats, E.; Barrio, M.; Tamarit, J.-L.; Crossley, S.; Li, W.; Pomjakushin, V.; Planes, A.; Mañosa, L.; Mathur, N.D.; et al. Giant barocaloric effects at low pressure in ferrielectric ammonium sulphate. Nat. Commun. 2015, 6, 8801. [CrossRef] [PubMed]

12. Khassaf, H.; Patel, T.; Alpay, S.P. Combined intrinsic elastocaloric and electrocaloric properties of ferroelectrics. J. Appl. Phys. 2017, 121, 144102. [CrossRef]

13. Liu, Y.; Wei, J.; Janolin, P.-E.; Infante, I.C.; Lou, X.; Dkhil, B. Giant room-temperature barocaloric effect and pressure-mediated electrocaloric effect in $\mathrm{BaTiO}_{3}$ single crystal. Appl. Phys. Lett. 2014, 104, 162904. [CrossRef]

14. Mikhaleva, E.A.; Flerov, I.N.; Bondarev, V.S.; Gorev, M.V.; Vasiliev, A.D.; Davydova, T.N. Electrocaloric and barocaloric effects in some ferroelectric hydrosulfates and triglycinesulfate. Ferroelectrics 2012, 430, 78-83. [CrossRef]

15. Gorev, M.V.; Mikhaleva, E.A.; Flerov, I.N.; Bogdanov, E.V. Conventional and inverse barocaloric effects in ferroelectric $\mathrm{NH}_{4} \mathrm{HSO}_{4}$. J. Alloys Compd. 2019, 806, 1047-1051. [CrossRef]

16. Landau, L.D.; Lifshitz, E.M. Statistical Physics, 3rd ed.; Butterworth-Heinemann: Oxford, UK, 1980; Volume 5.

17. Aleksandrov, K.S.; Flerov, I.N. Ranges of applicability of thermodynamic theory of structural phase transitions near the tricritical point. Sov. Phys. Solid State 1979, 21, 195-200.

18. Aleksandrov, K.S.; Beznosikov, B.V. Strukturnye Fazovie Perehody v Kristallah (Semeistvo Sulfata Kaliya); Nauka: Novosibirsk, Russia, 1993.

19. Mikhaleva, E.; Flerov, I.; Kartashev, A.; Gorev, M.; Bogdanov, E.; Bondarev, V. Thermal, dielectric and barocaloric properties of $\mathrm{NH}_{4} \mathrm{HSO}_{4}$ crystallized from an aqueous solution and the melt. Solid State Sci. 2017, 67, 1-7. [CrossRef]

20. Hoshino, S.; Vedam, K.; Okaya, Y.; Pepinsky, R. Dielectric and thermal study of $\left(\mathrm{NH}_{4}\right)_{2} \mathrm{SO}_{4}$ and $\left(\mathrm{NH}_{4}\right)_{2} \mathrm{BeF}_{4}$ transitions. Phys. Rev. 1958, 11, 405-412. [CrossRef]

21. Shmyt'ko, I.M.; Afonikova, N.S.; Torgashev, V.I. Anomalous states of the structure of $\left(\mathrm{NH}_{4}\right)_{2} \mathrm{SO}_{4}$ crystals in the temperature range 4.2-300 K. Phys. Solid State 2002, 44, 2309-2317. [CrossRef]

22. Kartashev, A.V.; Flerov, I.N.; Volkov, N.V.; Sablina, K.A. Adiabatic calorimetric study of the intense magnetocaloric effect and the heat capacity of $\left(\mathrm{La}_{0.4} \mathrm{Eu}_{0.6}\right)_{0.7} \mathrm{~Pb}_{0.3} \mathrm{MnO}_{3}$. Phys. Solid State 2008, 50, 2115-2120. [CrossRef]

23. Flerov, I.N.; Gorev, M.V.; Fokina, V.D.; Bovina, A.F.; Laptash, N.M. Calorimetric and X-ray diffraction studies of the $\left(\mathrm{NH}_{4}\right)_{3} \mathrm{WO}_{3} \mathrm{~F}_{3}$ and $\left(\mathrm{NH}_{4}\right)_{3} \mathrm{TiOF}_{5}$ perovskite-like oxyfluorides. Phys. Solid State 2004, 46, 915-921. [CrossRef]

24. O'Reilly, D.E.; Tsang, T. Order-disorder transition in ferroelectric ammonium sulfate. J. Chem. Phys. 1969, 50, 2274-2275. [CrossRef]

25. Gorev, M.V.; Flerov, I.N.; Bogdanov, E.V.; Voronov, V.N.; Laptash, N.M. Barocaloric effect near the structural phase transition in the $\mathrm{Rb}_{2} \mathrm{KTiOF}_{5}$ oxyfluoride. Phys. Solid State 2010, 52, 377-383. [CrossRef]

26. Kobayashi, J.; Enomoto, Y.; Sato, Y. A phenomenological theory of dielectric and mechanical properties of improper ferroelectric crystals. Phys. Stat. Solidi 1972, 50, 335-343. [CrossRef] 
27. Romanyuk, N.A.; Gaba, V.M.; Ursul, Z.M. Optic and temperature anomalies of ammonium sulfate in phase transitions. Ukr. Fiz. Zhurnal 1988, 33, 1381-1388.

28. Flerov, I.; Gorev, M.; Kartashev, A.; Pogorel'tsev, E.; Laptash, N. Heat capacity, thermal expansion and barocaloric effect in fluoride $\mathrm{K}_{2} \mathrm{TaF}_{7}$. J. Mat. Sci. 2019, 54, 14287-14295. [CrossRef]

(C) 2020 by the authors. Licensee MDPI, Basel, Switzerland. This article is an open access article distributed under the terms and conditions of the Creative Commons Attribution (CC BY) license (http:/ / creativecommons.org/licenses/by/4.0/). 